\title{
On the accretion flow geometry in $\mathrm{A} 0535+26$
}

\author{
N. R. Ikhsanov ${ }^{1,2}$, V. M. Larionov ${ }^{3,4}$, and N. G. Beskrovnaya ${ }^{2}$ \\ 1 Max-Planck-Institut für Radioastronomie, Auf dem Hügel 69, 53121 Bonn, Germany \\ 2 Central Astronomical Observatory of the Russian Academy of Science at Pulkovo, Pulkovo 65-1, \\ 196140 Saint-Petersburg, Russia \\ 3 Astronomical institute of St. Petersburg University, St. Petersburg, Russia \\ ${ }^{4}$ Isaac Newton Institute of Chile, St. Petersburg Branch, Russia
}

Received 28 November 2000 / Accepted 20 March 2001

\begin{abstract}
The geometry of accretion flow in the Be/X-ray transient A0535+26 is explored. It is shown that neither moderate nor giant X-ray flaring events observed in the system can be interpreted within the spherically symmetrical accretion model and hence the formation of an accretion disk around the neutron star magnetosphere during the both types of flares is required. The accretion disk can be formed at the periastron if (i) the expansion velocity of the Be star envelope in the equatorial plane is $V_{\mathrm{wr}}<150 \mathrm{~km} \mathrm{~s}^{-1}$ and (ii) the parameter accounting for the accretion flow inhomogeneities, $\xi$, satisfies the following condition: $\xi \gtrsim 0.16 \dot{M}_{17}^{-1 / 7}$, where $\dot{M}_{17}$ is the rate of mass capture by the neutron star expressed in units of $10^{17} \mathrm{~g} \mathrm{~s}^{-1}$. We suggest that the "missing" outburst phenomenon can be associated with the spherically symmetrical accretion onto the interchange-stable magnetosphere of the neutron star. The average spin up rate of the neutron star during moderate flares $\lesssim 3.510^{-12} \mathrm{~Hz} \mathrm{~s}^{-1}$ is predicted.
\end{abstract}

Key words. accretion - magnetic fields - stars: close binaries - stars: Be - stars: neutron star

\section{Introduction}

A $0535+26$ is a close binary system which contains the O9.7 IIe-B0 IIIe star HDE 245770 and a rotating, strongly magnetized neutron star. The optical companion is the source of intensive stellar wind $\left(\sim 10^{-8} M_{\odot} \mathrm{yr}^{-1}\right)$, which consists of a high velocity, low density component at high latitudes and a low velocity high density circumstellar disk at the equatorial plane. The star is observed to be variable in the optical-IR on time scales from a few months to a few years (Giovannelli \& Graziati 1992; Haigh et al. 1999).

A $0535+26$ was discovered as a source of hard X-ray emission by Ariel V during the giant outburst in 1975 (Rosenberg et al. 1975; Coe et al. 1975). Further observations have shown this system to be a transient X-ray source in which the neutron star (a $103 \mathrm{~s}$ pulsar with the surface magnetic field of $B \approx 10^{13}$ G: Kanno 1980; Dal Fiume et al. 1988) is in an eccentric orbit $(e=0.47 \pm 0.02)$ of the $P_{\text {orb }} \simeq 110.3 \pm 0.3$ days period around the Be companion (Finger et al. 1996). On the time scale of $P_{\text {orb }}$ the system may exhibit either no outburst ("missing" outburst), a moderate ("normal") or a giant outburst. Almost all moderate X-ray outbursts occur around a certain orbital phase. The X-ray flux at the maximum of moderate

Send offprint requests to: N. R. Ikhsanov, e-mail: ikhsanov@mpifr-bonn.mpg.de flares lies within the interval $0.1 \div 0.8 \mathrm{Crab}$ (in the 2 $10 \mathrm{keV}$ band) and their duration is $\sim 10 \div 15$ days. Giant $\mathrm{X}$-ray flares are of longer duration (up to 40 days) and the maximum X-ray flux detected during these events exceeds that of moderate flares by almost an order of magnitude. Some of giant flares ${ }^{1}$ are delayed in phase with respect to the moderate flares by 10-15 days. The spin up behaviour of the neutron star and the quasi periodic oscillations (QPOs) have been reported for the giant flare 1994 (Finger et al. 1996 and references therein).

The commonly accepted interpretation of the X-ray transient behaviour of A0535+26 is based on the so called eccentric orbit model. According to this model the rate of mass capture by the neutron star from the stellar wind of the normal companion depends on the orbital phase. It reaches its maximum value during the periastron passage when the neutron star moves through the dense equatorial disk of the Be star. As a result, all X-ray flares are expected to occur at the same orbital phase, which is associated with the periastron, $\phi=0.0$ (see Giovannelli \& Graziati 1992).

Some effort has been made to explain the difference in observed properties of the moderate and giant flares.

\footnotetext{
${ }^{1}$ Since 1975 five giant flares have been observed in the system.
} 
De Loore et al. (1984) suggested that giant flares occur due to temporal enhancement of plasma density in the vicinity of the neutron star due to the Be star envelope ejection. Another possibility has been discussed by Motch et al. (1991) who pointed out that moderate and giant flares can be triggered by different accretion mechanisms: spherical and disk accretion, respectively. Finally, Negueruela et al. (1998) associated giant flares with large-scale perturbations in the tidally truncated circumstellar disk of the Be companion.

The phenomenon of "missing" flares remains one of the most puzzling points in the interpretation of A0535+26. First it was assumed that the absence of X-ray outburst at the orbital phase $\phi=0.0$ is caused by the lower Be star activity and, correspondingly, the decrease of plasma density in the stellar envelope (Giovannelli \& Graziati 1992). However, systematic multiwavelength studies of the system reported recently (Clark et al. 1998a and references therein) did not confirm this hypothesis: "missing" flare phenomena occured during the period when the opticalIR flux of the star was increasing.

In this paper we explore a possibility to explain the observed diversity of X-ray flaring in A0535+26 in terms of variable geometry of accretion flow onto the magnetosphere of the neutron star. In the next section the spherically symmetrical accretion onto the neutron star magnetosphere is discussed. We find that in the particular case of A $0535+26$ the magnetospheric boundary is stable with respect to interchange instabilities. The upper limit to X-ray luminosity in this case is two orders of magnitude smaller than the X-ray luminosity observed during the moderate flares. On this basis, we suggest that the accretion flow during the X-ray flares in the system has a disk-like geometry. The condition for the accretion disk formation is discussed in Sect. 3. Application of our approach to the interpretation of the "missing" outburst phenomenon is the subject of Sect. 4. In Sect. 5 we examine possible observational signs of an accretion disk during moderate flares. The results are summarized in Sect. 6 .

\section{Spherically symmetrical accretion}

Within the spherically symmetrical accretion model, the plasma captured by the neutron star from the wind of the Be companion is assumed to flow toward the compact star in an almost radial direction with the free-fall velocity,

$V_{\mathrm{ff}}(R)=\sqrt{2 G M_{\mathrm{ns}} / R}$

Interaction of the accreting plasma with the magnetic field of the neutron star leads to the formation of a magnetosphere that, to a first approximation, prevents the plasma from reaching the star surface. In this context, the mode by which the accreting plasma enters the magnetosphere proves to be a key question in the interpretation of the accretion-powered sources.
As shown in a previous paper (Ikhsanov 2001) the rate of plasma penetration into the magnetosphere due to diffusion process,

$$
\dot{M}_{\text {diff }} \sim 10^{12} \mathrm{~g} \mathrm{~s}^{-1} \zeta^{1 / 2} \mu_{31}^{-1 / 14} M_{1.5}^{1 / 7}\left(\frac{\dot{M}_{\mathrm{c}}}{10^{17} \mathrm{~g} \mathrm{~s}^{-1}}\right)^{11 / 14}
$$

is too small to explain the observed X-ray luminosity of the system. Here, $\mu_{31}$ and $M_{1.5}$ are the magnetic moment and mass of the neutron star expressed in units of $10^{31} \mathrm{G} \mathrm{cm}^{3}$ and $1.5 M_{\odot}$, respectively, $\zeta$ is the efficiency of the diffusion process $(\zeta<1)$ and $\dot{M}_{\mathrm{c}}$ is the mass of the surrounding material with which the neutron star moving through the wind of the Be companion interacts in a time unit (see Sect. 4).

If the penetration process is governed by the reconnection of the field lines, the mass accretion rate onto the surface of the neutron star in A0535+26 can be evaluated as follows

$$
\dot{M}_{\text {rec }} \simeq 10^{15} \mathrm{~g} \mathrm{~s}^{-1}\left[\frac{\alpha_{\mathrm{R}}}{0.1}\right]\left[\frac{\lambda_{\mathrm{m}}}{0.1 R_{\mathrm{m}}}\right]\left(\frac{\dot{M}_{\mathrm{c}}}{10^{17} \mathrm{~g} \mathrm{~s}^{-1}}\right),
$$

where $\alpha_{\mathrm{R}}$ is the efficiency of the reconnection process and $\lambda_{\mathrm{m}}$ is the characteristic scale of the magnetic field in the accretion flow. As has been recently shown by Ikhsanov (2001), the reconnection-driven accretion model allows one to interpret the quiescent X-ray emission observed from A0535+26. At the same time, for the same accretion mechanism to be responsible for the system X-ray emission during flares, the number density of plasma in the inner radius of the Be star circumstellar disk should be in excess of $\sim 10^{13} \mathrm{~cm}^{-3}$. This value, however, is rather non-realistic since it exceeds by an order of magnitude the number density of plasma in the disk evaluated by Clark et al. (1998b).

The rate of plasma entry into the magnetosphere could be higher if the magnetospheric boundary is unstable with respect to interchange instabilities. However, the application of this scenario to the particular case of A0535+26 encounters a serious problem.

Arons \& Lea (1976) and Elsner \& Lamb (1976) have shown that the magnetospheric boundary of a neutron star undergoing spherical accretion is convex towards the accreting plasma. That is why the gradient of the field is such as to stabilize the boundary. In this situation, the magnetosphere is interchange unstable if the effective gravitational acceleration at the boundary has a positive sign:

$g_{\mathrm{eff}}=\frac{G M_{\mathrm{ns}}}{R_{\mathrm{m}}^{2}(\kappa)} \cos \kappa-\frac{V_{\mathrm{T}_{\mathrm{i}}}^{2}\left(R_{\mathrm{m}}\right)}{R_{\mathrm{c}}(\kappa)}>0$,

where $R_{\mathrm{c}}$ is the curvature radius of the field lines, $\kappa$ is the angle between the radius vector and the outward normal to the magnetospheric boundary and $V_{\mathrm{T}_{\mathrm{i}}}\left(R_{\mathrm{m}}\right)$ is the ion thermal velocity of the accreting plasma at the boundary.

This condition can be satisfied if the cooling time of plasma at the boundary is smaller than the free-fall time, i.e. a typical time of plasma heating due to accretion. 
According to Arons \& Lea and Elsner \& Lamb this can be realized only if the luminosity of the X-ray source is

$$
\begin{aligned}
L_{\mathrm{x}} \gtrsim L_{\mathrm{cr}} & \simeq 6.5 \times 10^{36} \mathrm{erg} \mathrm{s}^{-1} \\
& \times\left[\frac{\mu}{10^{31} \mathrm{G}}\right]^{1 / 4}\left[\frac{M_{\mathrm{ns}}}{1.5 M_{\odot}}\right]^{1 / 2}\left[\frac{R_{\mathrm{ns}}}{10^{6} \mathrm{~cm}}\right]^{-1 / 8}
\end{aligned}
$$

and thus, the Compton cooling is effective. Other mechanisms, like free-free and/or cyclotron cooling, are not effective in this case because of the relatively low density of the accretion flow beyond the magnetospheric boundary and the insufficiently high strength of the magnetic field at the Alfvén surface, respectively.

The condition $L_{\mathrm{x}} \gtrsim L_{\mathrm{cr}}$ in the case of $\mathrm{A} 0535+26$ is satisfied only during giant flares and at the maximum of a few moderate flares. Excluding these events, the instabilities of the boundary are suppressed and the rate of plasma penetration into the magnetic field of the neutron star is limited by $\dot{M}_{\text {rec }}$. This makes the interpretation of flares with luminosity $L_{\mathrm{x}}<6 \times 10^{36} \mathrm{erg} \mathrm{s}^{-1}$ in the frame of the spherical accretion approach very problematic. Furthermore, for the effective penetration of plasma into the star magnetosphere, the luminosity of the X-ray source should already be above the critical value $L_{\mathrm{cr}}$. This situation could be realized if an additional process operates which leads to periodical ( $\sim 11$ days) outbursts with luminosity $L_{\mathrm{x}} \gtrsim 7 \times 10^{36} \mathrm{erg} \mathrm{s}^{-1}$ inside the neutron star magnetosphere. However, extensive observations of A $0535+26$ have shown no evidence for such a process. On this basis, we focus on the investigation of another possibility. Here we explore whether the observed transient X-ray flaring in A $0535+26$ can be explained in terms of deviations of accretion flow geometry from the spherically symmetric case.

\section{Transient disk formation}

The problem of accreting plasma penetration into the magnetic field of the neutron star described in the previous section can be avoided if the accretion disk forms around the magnetosphere. As shown by Ghosh \& Lamb (1979), the rate of plasma penetration from the accretion disk into the magnetosphere of a neutron star is large enough to power the X-ray emission of the brightest $\mathrm{X}$-ray pulsars. In this context the question about the effective penetration of the accreting plasma into the neutron star magnetosphere is reduced to the question about the conditions for accretion disk formation in the system.

\subsection{Wind-fed mass transfer}

The Roche lobe radius of the Be component can be estimated following Eggleton (1983), taking the mass ratio $q=M_{\mathrm{Be}} / M_{\mathrm{ns}}=10$ as

$R_{\mathrm{L}_{\mathrm{Be}}} \simeq 3.4 \times 10^{12} \mathrm{~cm}\left(\frac{a_{0}}{8.6 \times 10^{12} \mathrm{~cm}}\right)$, where $a_{0}$ is the orbital separation at the periastron ${ }^{2}$. Hence, the ratio of the radius of the optical companion to its mean Roche lobe radius in $\mathrm{A} 0535+26$ is

$\frac{R_{\mathrm{Be}}}{R_{\mathrm{L}_{\mathrm{Be}}}} \lesssim 0.3\left(\frac{R_{\mathrm{Be}}}{14 R_{\odot}}\right)\left(\frac{R_{\mathrm{L}_{\mathrm{Be}}}}{3.4 \times 10^{12} \mathrm{~cm}}\right)^{-1}$.

This clearly indicates that the optical component underfills its Roche lobe and thus, the wind-fed mass transfer is realized in the system.

\subsection{Condition for an accretion disk formation}

For a disk to form, the specific angular momentum of matter captured by the compact star must be sufficient to allow it to enter a Keplerian circular orbit around the magnetosphere:

$R_{\mathrm{m}}<R_{\mathrm{circ}} \simeq \frac{\dot{J}^{2}}{\dot{M}_{\mathrm{c}}^{2} G M_{\mathrm{ns}}}$,

where $R_{\text {circ }}$ is the circularization radius and $\dot{J}$ is the rate of accretion of angular momentum, which is

$\dot{J}=\xi \dot{J}_{0}=\xi\left(\frac{1}{2} \Omega_{\mathrm{orb}} r_{0}^{2} \dot{M}_{\mathrm{c}}\right)$.

Here, $\Omega_{\text {orb }}$ and $r_{0}$ are the orbital angular velocity and the accretion radius of the neutron star, respectively. Parameter $\xi$ is the factor by which the average rate of accretion of angular momentum is reduced due to inhomogeneities (the velocity and density gradients) in the accretion flow.

If a neutron star is a component of a close binary system, its accretion radius is

$r_{0} \simeq\left\{\begin{array}{lll}r_{\alpha}=2 G M_{\mathrm{ns}} / V_{\text {rel }}^{2}, & \text { for } & V_{\text {rel }}>V_{0}, \\ R_{\mathrm{L}_{\mathrm{ns}}}, & \text { for } & V_{\text {rel }} \lesssim V_{0},\end{array}\right.$

where $R_{\mathrm{L}_{\mathrm{ns}}}$ is the neutron star Roche lobe radius and the velocity $V_{0}$ is determined as $r_{\alpha}\left(V_{0}\right)=R_{\mathrm{L}_{\mathrm{ns}}}$. Using the parameters of $\mathrm{A} 0535+26$, we find at the periastron point

$R_{\mathrm{L}_{\mathrm{ns}}} \simeq 1.8 \times 10^{12} \mathrm{~cm}\left(\frac{a_{0}}{8.6 \times 10^{12} \mathrm{~cm}}\right)$,

and the value of $V_{0}$ :

$V_{0}=150 \mathrm{~km} \mathrm{~s}^{-1}\left[\frac{M_{\mathrm{ns}}}{1.5 M_{\odot}}\right]^{1 / 2}\left[\frac{a_{0}}{8.6 \times 10^{12} \mathrm{~cm}}\right]^{-1 / 2}$.

The parameter $\xi$ has been evaluated from the numerical simulations of mass transfer in wind-fed close binaries (e.g. Anzer et al. 1987; Taam \& Fryxell 1988; Matsuda et al. 1991). These studies revealed the parameter $\xi$ to be variable, due to flip-flop instability of the accretion flow on a time scale of a few $\times r_{0} / V_{\mathrm{ff}}\left(r_{0}\right)$ with an average value $\bar{\xi}=0.2$. Combining this result with Eqs. $(6-10)$ we find

\footnotetext{
${ }^{2}$ Here we assume the eccentricity of the orbit to be $e=0.5$.
} 
the conditions for the accretion disk formation at the periastron in A0535+26 in the following form

$$
\left\{\begin{array}{llll}
V_{\text {rel }}<160 \mathrm{~km} \mathrm{~s}^{-1} \xi_{0.2}^{1 / 4} \dot{M}_{17}^{1 / 28} & \text { for } & V_{\text {rel }}>V_{0}, \\
\xi \gtrsim 0.16 \dot{M}_{17}^{-1 / 7} & \text { for } & V_{\text {rel }} \lesssim V_{0} .
\end{array}\right.
$$

On this basis the following conclusions on the flaring scenario in $\mathrm{A} 0535+26$ can be made.

\section{4. "Missing" flare phenomenon}

The average mass capture rate by the neutron star at the periastron in A0535+26 can be evaluated as follows

$\dot{M}_{\mathrm{c}}\left(a_{0}\right)=\pi r_{0}^{2}\left(a_{0}\right) \rho\left(a_{0}\right) V_{\text {rel }}\left(a_{0}\right)$,

where $\rho\left(a_{0}\right)$ is the density of plasma surrounding the neutron star at the periastron.

Following Lamers \& Waters (1987), the density and velocity in the circumstellar disk can be expressed as

$\left[\begin{array}{l}\rho(R)=\rho_{0}\left(R / R_{\mathrm{Be}}\right)^{-n}, \\ V_{\mathrm{wr}}(R)=V_{\mathrm{r} 0}\left(R / R_{\mathrm{Be}}\right)^{n-2},\end{array}\right.$

where $\rho_{0}$ and $V_{\text {ro }}$ are the density and velocity of plasma at the inner disk radius and the value of $n$ lies within the interval $2.3 \div 3.3$. For the case of $\mathrm{A} 0535+26$, we set hereafter $n=3$ (for a discussion see Clark et al. 1999).

Investigating the properties of Paschen emission lines in A0535+26, Clark et al. (1998b) have estimated the plasma density in the circumstellar disk to be $10^{12} \mathrm{~cm}^{-3}$. Assuming these lines to be generated in the inner part of the disk one finds $\rho_{0}=(1 \div 2) \times 10^{-12} \mathrm{~g} \mathrm{~cm}^{-3}$.

The value of $V_{\text {r0 }}$ suggested by Lamers \& Waters (1987) for field Be stars lies within the interval $2-20 \mathrm{kms}^{-1}$. Applying this to A0535+26 we find the mass outflow rate in the disk of $(1 \div 10) \times 10^{-8} M_{\odot} \mathrm{yr}^{-1}$, which is in good agreement with that estimated by Clark et al. $(1999)^{3}$.

Finally, the relative velocity can be presented in the following form

$\boldsymbol{V}_{\mathrm{rel}}=\boldsymbol{V}_{\mathrm{ns}}+\boldsymbol{V}_{\mathrm{w}}$,

where $\boldsymbol{V}_{\mathrm{ns}}$ is the linear velocity of the neutron star orbiting around the Be companion and $\boldsymbol{V}_{\mathrm{w}}$ is the stellar wind velocity in the frame of the Be star:

$\boldsymbol{V}_{\mathrm{w}}=V_{\mathrm{wr}} \boldsymbol{e}_{\mathrm{r}}+V_{\mathrm{w} \phi} \boldsymbol{e}_{\phi}$.

Here $\boldsymbol{e}_{\mathrm{r}}$ and $\boldsymbol{e}_{\phi}$ are the unit vectors in the radial and azimuthal directions, respectively. At the periastron, one can set $^{4}$

$V_{\mathrm{ns}}\left(a_{0}\right)=\frac{2 \pi \bar{a}}{P_{\mathrm{orb}}} \sqrt{\frac{1+e}{1-e}}$,

$V_{\mathrm{w} \phi}\left(a_{0}\right) \lesssim \sqrt{G M_{\mathrm{Be}} / a_{0}}$,

\footnotetext{
${ }^{3}$ Here we adopted the disk opening angle of $\theta=15^{\circ}$.

${ }^{4}$ Here we take into account that $M_{\mathrm{ns}} \ll M_{\mathrm{Be}}$.
}

where $\bar{a}$ is the average value of the orbital separation.

Thus, using these values in Eq. (12) and calculating $r_{0}$ from Eq. (8) one can express the mass capture rate by the neutron star during the periastron passage as

$$
\begin{aligned}
\left.\dot{M}_{\mathrm{c}}\left(a_{0}\right)\right|_{\left(V_{\mathrm{rel}} \gtrsim V_{0}\right)} \simeq 10^{17} \mathrm{~g} \mathrm{~s}^{-1} M_{1.5}^{2} \\
\times\left(\frac{N\left(a_{0}\right)}{10^{9} \mathrm{~cm}^{-3}}\right)\left(\frac{V_{\mathrm{rel}}\left(a_{0}\right)}{180 \mathrm{~km} \mathrm{~s}^{-1}}\right)^{-3},
\end{aligned}
$$

and

$$
\begin{array}{r}
\left.\dot{M}_{\mathrm{c}}\left(a_{0}\right)\right|_{\left(V_{\mathrm{rel}}<V_{0}\right)} \simeq 7 \times 10^{16} \mathrm{~g} \mathrm{~s}^{-1} M_{1.5}^{2} \\
\times\left(\frac{N\left(a_{0}\right)}{10^{9} \mathrm{~cm}^{-3}}\right)\left(\frac{V_{\mathrm{rel}}\left(a_{0}\right)}{40 \mathrm{~km} \mathrm{~s}^{-1}}\right) .
\end{array}
$$

On the other hand, the rate of mass accretion onto the neutron star surface during the missing flare phenomenon is at least an order of magnitude smaller than the mass capture rate evaluated above. Hence, if the absence of a $\mathrm{X}$-ray outburst at the periastron is caused by the lower activity of the Be star, the density of the circumstellar disk during this time should be smaller than its average value by at least a factor of 10 . Under this condition, the disk is expected to be faint or almost invisible.

However, systematic optical-IR observations have shown the "missing" flare phenomena to occur also during the periods when the circumstellar disk is in its brightest state (see Clark et al. 1998a). This suggests that the low activity of the Be star is not the only reason for the "missing" flare phenomenon and an additional factor, which leads to suppression of X-ray flaring at the periastron, operates in the system.

Within the approach presented in this paper, the "missing" flare phenomenon can be associated with the spherical geometry of the accretion flow and, correspondingly, the low rate of plasma penetration into the magnetosphere of the neutron star. According to condition (11) this situation is realized if either the relative velocity exceeds the critical value or if the parameter $\xi$ is small enough to prevent the formation of the accretion disk around the neutron star magnetosphere.

Combining Eqs. (14-17) we find that an accretion disk around the neutron star cannot form if the radial velocity of plasma in the circumstellar disk of the Be companion is $V_{\mathrm{wr}}\left(a_{0}\right) \gtrsim 150 \mathrm{~km} \mathrm{~s}^{-1}$. Under this condition the mass accretion rate onto the neutron star surface is limited by $\dot{M}_{\text {rec }}$ and thus, the X-ray luminosity of the neutron star during the periastron passage remains almost at the quiescent level.

In the case of $V_{\mathrm{wr}}\left(a_{0}\right)<150 \mathrm{~km} \mathrm{~s}^{-1}$, the missing flare phenomenon can occur only if the second part of condition (11) is not satisfied. Analysis of this situation is especially important if the Be companion of $\mathrm{A} 0535+26$ is surrounded by the viscous decretion disk (Lee et al. 1991; Porter 1999). In this case the radial plasma velocity at the periastron is expected to be relatively small, i.e. only a few $\times \mathrm{kms}^{-1}$ (Hanuschik 2000; Okazaki 2000). In this 
situation Eq. (15) can be simplified, neglecting the term $V_{\mathrm{wr}}$ that gives the value of the relative velocity at the periastron, $V_{\text {rel }}\left(a_{0}\right) \simeq 4 \times 10^{6} \mathrm{~cm} \mathrm{~s}^{-1}$ and, correspondingly, the strength of the stellar wind $\dot{M}_{\mathrm{c}}\left(a_{0}\right) \simeq 7 \times 10^{16} \mathrm{~g} \mathrm{~s}^{-1}$. Under these conditions the formation of the accretion disk around the neutron star in A0535+26 does not occur if $\xi \lesssim 0.17$, i.e. if the parameter $\xi$ is slightly smaller than its average value $\bar{\xi}$. According to Taam \& Fryxell (1988), the variations of the parameter $\xi$ in the wind-fed massexchange close binaries is not unusual. In particular, they found the value of $\xi$ to vary due to flip-flop instability within the interval $0.05 \lesssim \xi<1$. The characteristic time of this instability in the case of A $0535+26$ is

$t_{\mathrm{var}} \simeq$ a few $\times \frac{r_{0}^{3 / 2}}{\sqrt{2 G M_{\mathrm{ns}}}} \sim 4 \div 7$ days.

Since $t_{\mathrm{var}}$ exceeds the time interval in which the moderate flares are scattered around the zero orbital phase $(\Delta \phi \approx$ $0.02 \sim 2$ days) one can envisage a situation in which the value of $\xi$ at the periastron proves to be small enough for the condition (11) not to be satisfied. If this happens, the geometry of the accretion flow is spherically symmetrical and the mass accretion rate onto the neutron star surface is limited by $\dot{M}_{\text {rec }}$.

\section{Possible signs of accretion disk during moderate flares}

The last question we briefly address in this paper is the observational appearance of the accretion disk in A0535+26 during moderate flares.

The conclusion about the presence of an accretion disk in the system during giant flares has been made on the basis of X-ray observations of the neutron star spin up behaviour and the QPOs (see Finger et al. 1996 and references therein). Can these criteria be used to conclude the presence or absence of the accretion disk during moderate flares? up if

A neutron star undergoing disk accretion is spinning

$\frac{\mathrm{d} I \omega}{\mathrm{d} t}=K_{\mathrm{su}}-K_{\mathrm{sd}}>0$,

where $I$ is the moment of inertia of the neutron star, $\omega=2 \pi / P_{\mathrm{s}}$ and $K_{\mathrm{su}}$ and $K_{\mathrm{sd}}$ are the accelerating and decelerating torques applied to the neutron star, respectively.

Following Lipunov (1992) we take

$K_{\mathrm{su}} \approx \dot{M}_{\mathrm{c}} \sqrt{G M_{\mathrm{ns}} R_{\mathrm{m}}}$

$K_{\mathrm{sd}} \approx k_{\mathrm{t}} \mu^{2} / R_{\mathrm{cor}}^{3}$,

where $k_{\mathrm{t}}$ is the dimensionless parameter of the order of unity and $R_{\text {cor }}$ is the corotational radius, which in the case of the neutron star in $\mathrm{A} 0535+26$ is

$R_{\text {cor }} \simeq 3.8 \times 10^{9} P_{103}^{2 / 3}\left[\frac{M_{\mathrm{ns}}}{1.5 M_{\odot}}\right]^{1 / 3} \mathrm{~cm}$.
Putting Eqs. (22) into (21) we find that the neutron star in A0535+26 is spinning up if the X-ray luminosity in a flare is

$$
L_{\mathrm{x}} \gtrsim 5 \times 10^{35} \mu_{31}^{2} R_{6} P_{103}^{-7 / 3}\left[\frac{M_{\mathrm{ns}}}{1.5 M_{\odot}}\right]^{-2 / 3} \mathrm{erg} \mathrm{s}^{-1} .
$$

This indicates that, in the frame of our approach, the neutron star is expected to be spinning up in both the giant and moderate flares. The spin up rate is $\dot{P} \propto L_{\mathrm{x}}^{6 / 7}$. Hence, taking as the basis the spin up observations of the flare 1994 (Finger et al. 1996), we can predict the spin up rate during moderate flares to be

$\dot{\nu}_{\mathrm{fm}} \simeq 3.5 \times 10^{-12} \mathrm{~Hz} \mathrm{~s}^{-1}\left[\frac{F_{(2-10 \mathrm{keV})}}{0.5 \mathrm{Crab}}\right]^{6 / 7}$,

where $F_{(2-10 \mathrm{keV})}$ is the X-ray flux during a moderate flare in the $2-10 \mathrm{keV}$ band.

The question whether it is possible to observe QPOs during moderate flares is more complicated. The answer depends on whether the accretion disk forming during a particular event is optically thick or thin. The detailed investigation of this question is beyond the scope of the present paper. Here we would like only to note that the modeling of a developed accretion disk during moderate flares faces some problems. In particular, assuming all material stored in the disk to be accreted onto the neutron star surface one can evaluate the mass of the disk as

$M_{\mathrm{d}} \simeq 5 \times 10^{20} \mathrm{~g} M_{1.5}^{-1} R_{6}\left[\frac{E_{\mathrm{f}}}{10^{41} \mathrm{erg}}\right]$,

where $E_{\mathrm{f}}$ is the total amount of energy released during the flare. This, however, is essentially smaller than the average mass of developed accretion disks estimated from observations of other close X-ray binaries (see Lipunov 1992). Furthermore, within the same assumption, the duration of a flare depends on the radial velocity of plasma in the disk. This means that the flare duration can be limited as

$\Delta t_{\mathrm{f}}>\frac{R_{\text {out }}}{V_{\mathrm{dr}}}=\frac{m_{\mathrm{p}} \sqrt{G M_{\mathrm{ns}} R_{\text {out }}}}{\alpha k T\left(R_{\mathrm{out}}\right)}$.

Here $T_{\mathrm{d}}\left(R_{\text {out }}\right)$ is the plasma temperature at the outer radius of the disk, $R_{\text {out }}$, and $V_{\mathrm{dr}}$ is the radial velocity of plasma in the accretion disk which according to Shakura \& Sunyaev (1973) is

$V_{\mathrm{dr}} \simeq \alpha c_{\mathrm{s}}^{2} / V_{\mathrm{k}}$,

where $c_{\mathrm{s}}$ is the sound speed and $V_{\mathrm{k}}$ is the keplerian velocity. If the disk is optically thick, i.e.

$T_{\mathrm{d}}\left(R_{\text {out }}\right)=\left(\frac{\dot{M}_{\mathrm{c}} G M_{\mathrm{ns}}}{4 \pi \sigma_{\mathrm{sb}} R_{\text {out }}^{3}}\right)^{1 / 4}$,

the flare duration is

$\Delta t_{\mathrm{f}}>50 \mathrm{~d} \alpha_{0.1}^{-1} M_{1.5}^{1 / 14} \mu_{31}^{5 / 7} \dot{M}_{18}^{-17 / 28}\left[\frac{R_{\text {out }}}{10 R_{\mathrm{m}}}\right]^{5 / 4}$ 
The derived value is comparable with the duration of giant flares but essentially exceeds the typical duration of moderate flares. This demonstrates that if the accretion disk is formed during a moderate flare, its structure is somewhat different from that of developed optically thick disks and, therefore, the currently suggested models of QPOs (e.g. Alpar \& Shaham 1985; Lamb et al. 1985; van der Klis et al. 1987) cannot be applied without additional investigation.

\section{Conclusion}

Our main conclusion is that the accretion disk around the neutron star magnetosphere forms during both the moderate and giant X-ray flares in $\mathrm{A} 0535+26$. The grounds for this conclusion are the low rate of plasma penetration into the neutron star magnetic field during the spherically symmetrical accretion state that does not allow us to explain the observed X-ray luminosity of the system during outbursts. The low rate of plasma penetration into the magnetosphere is the result of the stability of the magnetospheric boundary with respect to interchange instabilities. The X-ray luminosity of the system within the reconnection-driven accretion model is comparable with the X-ray luminosity of A0535+26 observed the during quiescent state (for discussion see Ikhsanov 2001).

The formation of an accretion disk around the magnetosphere of the neutron star in A0535+26 can be expected during the periastron passage if the following conditions are satisfied: (i) the expansion velocity of the Be star envelope in the equatorial plane is $V_{\mathrm{wr}}<150 \mathrm{~km} \mathrm{~s}^{-1}$ and (ii) the parameter accounting for the accretion flow inhomogeneities (density and velocity gradients) is $\xi \gtrsim$ $0.16 \dot{M}_{17}^{-1 / 7}$, where $\dot{M}_{17}$ is the rate of mass capture by the neutron star from the stellar wind of the normal companion expressed in units of $10^{17} \mathrm{~g} \mathrm{~s}^{-1}$.

In the frame of the approach presented in this paper, we associate the "missing" flare phenomenon at the orbital phase $\phi=0$ with the spherical geometry of the accretion flow. The reasons for this can be high expansion velocity of the Be star envelope and/or a spontaneous decrease of the parameter $\xi$ slightly below its average value due to flip-flop instability of the accretion flow.

Finally, the presented approach predicts the average spin up of the neutron star during moderate flares of $\sim 3.5 \times 10^{-12} \mathrm{~Hz} \mathrm{~s}^{-1}$.

Acknowledgements. We would like to thank the referee for useful comments and suggesting improvements and A. E. Tarasov and V. M. Lyuty for helpful discussions. NRI acknowledge the support of the Follow-up program of the
Alexander von Humboldt Foundation. The work was partly supported by INTAS under the grant YSF 99-4004, RFBR under the grant 99-02-16336 and by the Federal program "INTEGRATION" under the grant KO 232.

\section{References}

Alpar, M. A., \& Shaham, J. 1985, Nature, 316, 239

Anzer, U., Börner, G., \& Monaghan, J. J. 1987, A\&A, 176, 235

Arons, J., \& Lea, S. M. 1976, ApJ, 207, 914

Clark, J. S., Tarasov, A. E., Steele, I. A., et al. 1998a, MNRAS, 294,165

Clark, J. S., Steele, I. A., Coe, M. J., \& Roche, P. 1998b, MNRAS, 297, 657

Clark, J. S., Lyuty, V. M., Zaitseva, G. V., et al. 1999, MNRAS, 302,167

Coe, M. J., Carpenter, G. F., Engel, A. R., \& Quenby, J. J. 1975, Nature, 256, 630

Dal Fiume, D., Frontera, F., \& Morelli, E. 1988, ApJ, 331, 313

de Loore, C., Giovannelli, F., van Dessel, E. L., et al. 1984, A\&A, 141, 279

Eggleton, P. P. 1983, ApJ, 268, 368

Elsner, R. F., \& Lamb, F. K. 1976, Nature, 262, 356

Finger, M. H., Wilson, R. B., \& Harmon, B. A. 1996, ApJ, 459, 288

Giovannelli, F., \& Graziati, L. S. 1992, Space Sci. Rev., 59, 1

Ghosh, P., \& Lamb, F. K. 1979, ApJ, 232, 259

Haigh, N. J., Coe, M. J., Steele, I. A., \& Fabregat, J. 1999, MNRAS, 310, L21

Hanuschik, R. W. 2000, in The Be Phenomenon in Early-type Stars, ed. M. Smith et al., ASP Conf. Ser., 214, 518

Ikhsanov, N. R. 2001, A\&A, 367, 549

Kanno, S. 1980, PASJ, 32, 105

Lamb, F. K., Shabazaki, N., Alpar, M. A., \& Shaham, J. 1985, Nature, 317, 681

Lamers, H. J. G. L. M., \& Waters, L. B. F. M. 1987, A\&A, 182,80

Lee, U., Osaki, Y., \& Saio, H. 1991, MNRAS, 250, 432

Lipunov, V. M. 1992, Astrophysics of neutron stars (Springer Verlag, Heidelberg)

Matsuda, T., Sekino, N., Sawada, K., et al. 1991, A\&A, 248, 301

Negueruela, I., Reig, P., Coe, M. J., \& Fabregat, J. 1998, A\&A, 336,251

Okazaki, A. T. 2000, in The Be Phenomenon in Early-type Stars, ed. M. Smith et al., ASP Conf. Ser., 214, 409

Motch, C., Stella, L., Janot-Pacheco, E., \& Mouchet, M. 1996, ApJ, 369, 490

Porter, J. M. 1999, A\&A, 348, 512

Rosenberg, F. D., Eyles, C. J., Skinner, G. K., \& Willmore, A. P. 1975 , Nature, 256, 628

Shakura, N. I., \& Sunyaev, R. A. 1973, A\&A, 24, 337

Taam, R. E., \& Fryxell, B. A. 1988, ApJ, 327, L73

van der Klis, M., Jansen, F., van Paradijs, J. P., et al. 1987, ApJ, 313, L19 